\title{
Fungos conidiais do bioma Caatinga II. Novos registros para o continente americano, Neotrópico, América do Sul e Brasil
}

Conidial fungi of Caatinga biome II. New records for American continent, Neotropics, South America and Brazil

Tasciano dos Santos Santa Izabel ${ }^{1,2}$, Dalila Souza Santos ${ }^{1}$, Davi Augusto Carneiro de Almeida ${ }^{1}$ \& Luís Fernando Pascholati Gusmão ${ }^{1}$

\begin{abstract}
Resumo
Durante investigação de fungos conidiais associados a materiais vegetais em decomposição em uma área de extrema relevância biológica no bioma Caatinga, município de Morro do Chapéu, estado da Bahia, novos registros para o continente Americano, América do Sul, Neotrópico e Brasil foram encontrados. Dendryphiopsis biseptata Morgan-Jones, R.C. Sinclair \& Eicker e Virgariella atra S. Hughes são novos registros para o continente Americano; Dictyochaeta matsushimae (Hewings \& J.L. Crane) Whitton, McKenzie \& K.D. Hyde, Endophragmiella boothii (M.B. Ellis) S. Hughes são reportados pela primeira vez para o Neotrópico; Anungitea palustris R.F. Castañeda \& W.B. Kendr., Dictyochaeta anamorfo de Chaetosphaeria dingleyae S. Hughes, W.B. Kendr. \& Shoemaker, Dictyochaeta anamorfo de Chaetosphaeria pulchriseta S. Hughes, W.B. Kendr. \& Shoemaker, Eversia parvula Hol.-Jech., Gyrothrix hughesii Piroz. e Minimelanolocus navicularis (R.F. Castañeda) R.F. Castañeda são novos registros para a América do Sul; Endophragmiella pallescens B. Sutton, Helicoubisia coronata Lunghini \& Rambelli e Selenodriella ponmudiensis (Varghese \& V.G. Rao) R.F. Castañeda \& Saikawa são reportados pela primeira vez para o Brasil. Descrições, comentários, distribuição geográfica e ilustrações são apresentados para estas espécies.

Palavras-chave: biodiversidade, fungos anamórficos, taxonomia.
\end{abstract}

\begin{abstract}
During investigation of conidial fungi from dead plant material in an area of extreme biological importance at Caatinga biome, municipality of Morro do Chapéu, Bahia State, Brazil, some new records for American continent, South America, Neotropics, and Brazil were found. Dendryphiopsis biseptata Morgan-Jones, R.C. Sinclair \& Eicker and Virgariella atra S. Hughes are new records for American continent; Dictyochaeta matsushimae (Hewings \& J.L. Crane) Whitton, McKenzie \& K.D. Hyde and Endophragmiella boothii (M.B. Ellis) S. Hughes are reported for the first time for Neotropics; Anungitea palustris R.F. Castañeda \& W.B. Kendr., Dictyochaeta anamorfic of Chaetosphaeria dingleyae S. Hughes, W.B. Kendr. \& Shoemaker, Dictyochaeta anamorfic of Chaetosphaeria pulchriseta S. Hughes, W.B. Kendr. \& Shoemaker, Eversia parvula Hol.-Jech., Gyrothrix hughesii Piroz. and Minimelanolocus navicularis (R.F. Castañeda) R.F. Castañeda are new records to South America; Endophragmiella pallescens B. Sutton, Helicoubisia coronata Lunghini \& Rambelli and Selenodriella ponmudiensis (Varghese \& V.G. Rao) R.F. Castañeda \& Saikawa are reported for the first time for Brazil. Description, comments, geographical distribution and illustrations are presented for these species.

Key words: anamorfic fungi, biodiversity, taxonomy.
\end{abstract}

\section{Introdução}

$\mathrm{Na}$ região semi-árida do Brasil o aspecto fitofisionômico predominante é a Caatinga, ocorrendo outros tipos vegetacionais como matas úmidas, matas estacionais, cerrados, tabuleiros e campos rupestres (Andrade-Lima 1981).
O conhecimento da diversidade de fungos conidiais na região semi-árida brasileira é bastante pontual (Gusmão et al. 2006). Maia \& Gibertoni (2002), em inventário da diversidade de fungos no semi-árido brasileiro, apresentaram uma listagem com 451 espécies distribuídas entre os Filos Ascomycota,

${ }^{1}$ Universidade Estadual de Feira de Santana, Depto. Ciências Biológicas, Lab. Micologia, Av. Transnordestina s/n, 44036-900, Feira de Santana, BA, Brasil.

${ }^{2}$ Autor para correspondência: tazuefsbot@gmail.com 
Basidiomycota, Oomycota, Zygomycota (incluindo a ordem Glomales, atualmente Glomeromycota) e os fungos conidiais, sendo estes representados por 198 espécies. Gusmão et al. (2006) através de uma compilação de dados chegaram ao número de 407 espécies de fungos conidiais no semi-árido.

Trabalhos publicados recentemente têm revelado novas espécies e novos registros destes fungos, ampliando consideravelmente o conhecimento da distribuição geográfica mundial de diversas espécies (Barbosa et al. 2007; Castañeda-Ruiz et al. 2006; Cruz et al. 2007a,b, 2008, 2009; Gusmão et al. 2008; LeãoFerreira et al. 2008; Marques et al. 2007).

Esse trabalho teve como objetivo caracterizar as espécies que constituem novos registros para o continente Americano, Neotrópico, América do Sul e Brasil, coletadas em áreas de Caatinga, campo rupestre e mata estacional que ocorrem no município de Morro do Chapéu, estado da Bahia.

\section{Materiais e Métodos}

O presente estudo foi realizado no município de Morro do Chapéu, situado ao Norte da Chapada Diamantina (10 $40^{\prime}-11^{\circ} 50^{\prime} \mathrm{S}$ e $\left.40^{\circ} 50^{\prime}-41^{\circ} 20^{\prime} \mathrm{W}\right)$, área considerada de extrema importância biológica (Velloso et al. 2002). A região possui uma tipologia vegetal única de Caatinga, que não se encontra representada em nenhuma das Unidades de Conservação no semi-árido (Maury 2002). Além da vegetação de Caatinga, apresenta outras formações vegetacionais como campo rupestre, florestas estacionais, vegetação de dunas interiores e áreas de transição (Junqueira \& Bianchini 2006).

Quatro expedições foram realizadas, entre maio de 2008 e fevereiro de 2009 , quando substratos vegetais em decomposição (cascas, folhas e galhos) foram coletados em seis pontos distintos: um de caatinga, um de campo rupestre e quatro de floresta estacional. Os substratos vegetais foram acondicionados em sacos de papel Kraft, transportados ao laboratório e submetidos à técnica de lavagem em água corrente (Castañeda-Ruiz 2005). Após secagem, as amostras foram acondicionadas em câmaras-úmidas. No período de 30 dias os substratos foram observados sob estereomicrocópio, sendo as estruturas de reprodução dos fungos coletadas e transferidas para meio de montagem permanente com resina PVL (álcool polivinílico + lactofenol). A identificação das espécies foi realizada comparando-se as estruturas reprodutivas encontradas com as descrições apresentadas em bibliografia especializada. O material foi depositado no Herbário da Universidade Estadual de Feira de Santana (HUEFS).

\section{Resultados e Discussão}

Foram encontradas 83 espécies de fungos conidiais, das quais duas constituem novos registros para o continente Americano, duas para o Neotrópico, seis para a América do Sul e três para o Brasil. A maioria das espécies foi coletada sobre folhas (51), seguido de cascas (28) e galhos (23). Quarenta e uma espécies ocorreram exclusivamente em folhas, 13 somente em cascas e 13 apenas em galhos. Três espécies ocorreram nos três substratos, seis ocorreram em folhas e cascas, uma em folhas e galhos e seis em cascas e galhos.

Anungitea palustris R.F. Castañeda \& W.B. Kendr., Univ. Waterloo Biol. Ser. 35: 10. 1991.

Fig. 1 a-d

Conidióforos macronemáticos, mononemáticos, simples, solitários ou em grupos, eretos, retos, septados, lisos, castanhos a castanho-claros, subhialinos no ápice, 34,5-127,5×4,5-6 $\mu \mathrm{m}$; células conidiogênicas poliblásticas, integradas, raramente evidentes, terminais, raramente laterais; dentículos proeminentes, 1-2 $\mu \mathrm{m}$ compr.; conídios catenulados, cilíndricos a fusiformes, secos, lisos, 1-septados, raramente 0 -septados, hialinos, 13,5-18 ×1,5-2,0 $\mu \mathrm{m}$. Material examinado: 12.II.2009, T.S. Santa Izabel s.n. (HUEFS 155095).

O gênero Anungitea $\mathrm{B}$. Sutton é caracterizado pelos conídios solitários ou formando cadeias simples, surgindo de dentículos conspícuos (Sutton 1973; Castañeda-Ruiz et al. 1997b). O gênero é composto por 13 espécies (Castañeda-Ruiz et al. 1997b). Anungitea palustris assemelha-se com A. fragilis $B$. Sutton pela morfologia do conídio, no entanto, essa espécie apresenta células conidiogênicas integradas, conídios castanho-claros e maiores (Sutton 1973). O material examinado está de acordo com a descrição apresentada por Castañeda-Ruiz \& Kendrick (1991). Este é o segundo registro da espécie para o mundo e o primeiro para a América do Sul. Estando presente também em Cuba (Castañeda-Ruiz \& Kendrick 1991).

Dendryphiopsis biseptata Morgan-Jones, R.C. Sinclair \& Eicker, Mycotaxon 17: 304. 1983.

Fig. 1 e-f

Conidióforos macronemáticos, mononemáticos, solitários ou em grupos, eretos, retos ou levemente flexuosos, ramificados no ápice, septados, lisos ou verrucosos, castanhos a castanho-escuros, 60-210 $\times$ 7,5-9 $\mu \mathrm{m}$; células conidiogênicas monotréticas, integradas, subglobosas, lisas, castanhas a 


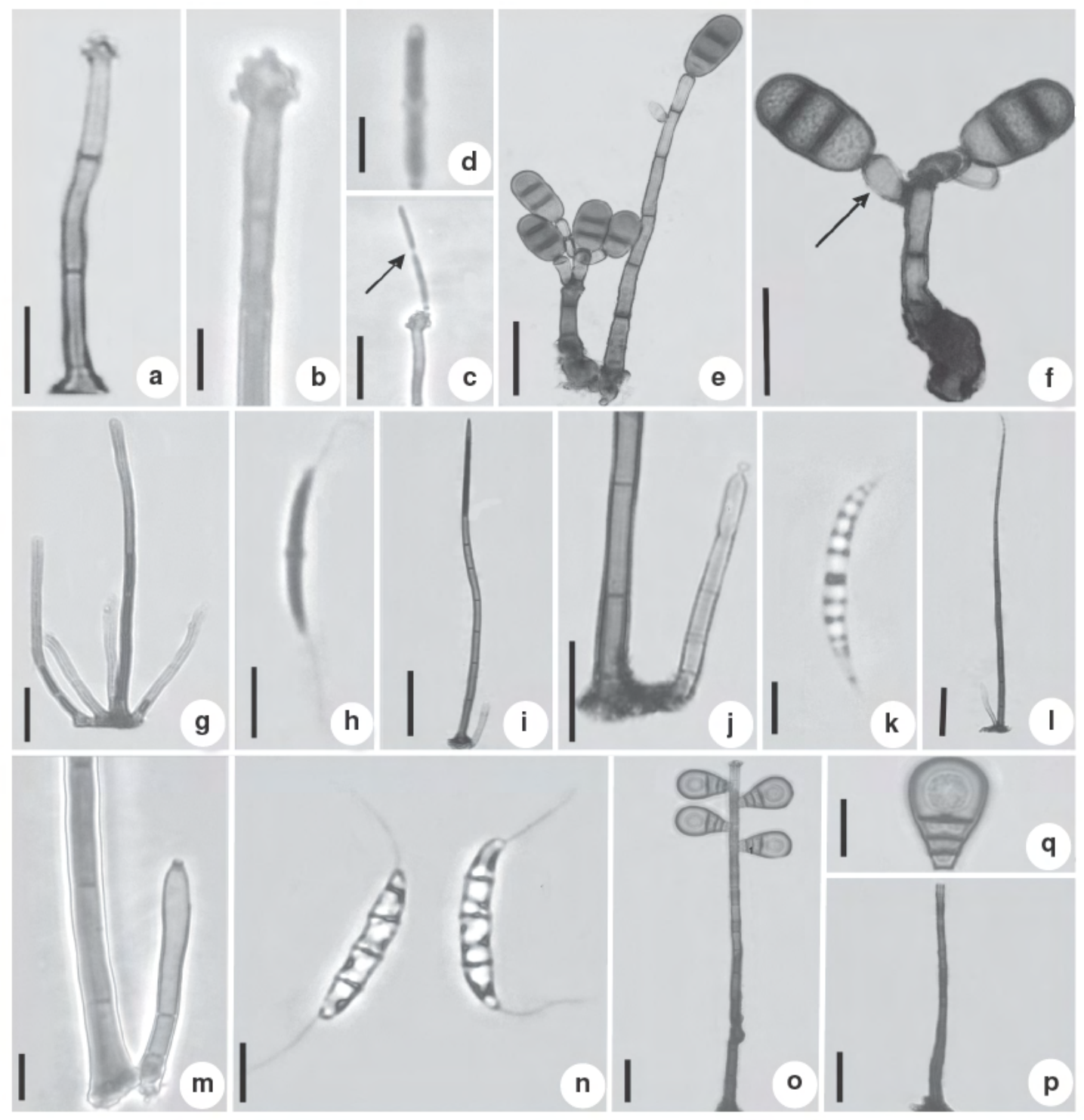

Figura 1 - a-d. Anungitea palustris R.F. Castañeda \& W.B. Kendr - a. aspecto geral; b. detalhe da célula conidiogênica; c. cadeia de conídios (seta); d. conídio. e-f. Dendryphiopsis biseptata Morgan-Jones, R.C. Sinclair \& Eicker-e. aspecto geral; f. detalhe da célula conidiogênica (seta). g-h. Dictyochaeta anamorfo de Chaetosphaeria dingleyae S. Hughes, W.B. Kendr - g. aspecto geral; h. conídio. i-k. Dictyochaeta anamorfo de Chaetosphaeria pulchriseta S. Hughes, W.B. Kendr - i. aspecto geral; j. detalhe do conidióforo; k. conídio. 1-n. Dictyochaeta matsushimae (Hewings \& J.L. Crane) Whitton - 1. aspecto geral; m. detalhe do conidióforo; n. conídios. o-q. Endophragmiella boothii (M.B. Ellis) S. Hughes - o. aspecto geral; p. conidióforo; q. conídio. Barra $=50 \mu$ (f, i, 1); $20 \mu(\mathrm{a}, \mathrm{c}, \mathrm{e}, \mathrm{g}, \mathrm{j}, \mathrm{o}, \mathrm{q}) ; 10 \mu(\mathrm{b}, \mathrm{m}, \mathrm{n}, \mathrm{p})$; $5 \mu(\mathrm{d}, \mathrm{h}, \mathrm{k})$.

Figure 1 - a-d. Anungitea palustris R.F. Castañeda \& W.B. Kendr - a. general aspect; b. detail of conidiogenous cell; c. catenate conidia (arrow); d. conidium. e-f. Dendryphiopsis biseptata Morgan-Jones, R.C. Sinclair \& Eicker - e. general aspect; f. detail of conidiogenous cell (arrow). g-h. Dictyochaeta anamorfic of Chaetosphaeria dingleyae S. Hughes, W.B. Kendr - g. general aspect; $h$ conidium. i-k. Dictyochaeta anamorfic of Chaetosphaeria pulchriseta S. Hughes, W.B. Kendr - i. general aspect; j. detail of conidiophore; k. conidium. 1-n. Dictyochaeta matsushimae (Hewings \& J.L. Crane) Whitton-1. general aspect; m. detail of conidiophore; n. conidia. o-q. Endophragmiella boothii (M.B. Ellis) S. Hughes - o. general aspect; p. conidiophore; q. conidium. Bar $=50 \mu$ (f, i, 1); $20 \mu$ (a, c, e, g, j, o, q); 10 $\mu$ (b, m, $\mathrm{n}, \mathrm{p}) ; 5 \mu$ (d, h, k). 
castanho-claras, 12-18×6-9 $\mu \mathrm{m}$; conídios solitários, secos, elipsóides a obovais, lisos, 2-septados, raramente 1-septados, castanhos, 33-42× 18-21 $\mu \mathrm{m}$. Material examinado: 28.XII.2007, D.S. Santos s.n. (HUEFS 134726).

Dendryphiopsis S. Hughes inclui cinco espécies (CABI 2010). Dendryphiopsis biseptata assemelha-se a D. atra (Corda) S. Hughes pela morfologia do conidióforo e do conídio; porém, distingue-se pelo número de septos nos conídios (Morgan-Jones et al. 1983). Dendryphiopsis biseptata foi descrito originalmente com conidióforos lisos. No entanto, no material estudado estes se apresentaram também verrucosos. Essa é a segunda ocorrência para o mundo e a primeira para o continente americano. Antes localizada na África do Sul (Morgan-Jones et al. 1983).

Dictyochaeta anamorfo de Chaetosphaeria dingleyae S. Hughes, W.B. Kendr. \& Shoemaker, N.Z. Jl. Bot. 6: 343. 1968.

Fig. $1 \mathrm{~g}$-h

Setas eretas, retas ou flexuosas, septadas, lisas, ápice arredondado, castanhas a castanho-escuras, castanho-clara no ápice, 97,5-150 × 5-5,5 $\mu \mathrm{m}$; conidióforos macronemáticos, mononemáticos, solitários ou em grupos de 2-4 associados a uma seta, eretos, retos ou ligeiramente flexuosos, septados, lisos, castanho-claros, 37,5-73,5 × 2,43,6 $\mu \mathrm{m}$; células conidiogênicas monofialídicas, integradas, terminais, lisas, determinadas, com colaretes proeminentes; conídios agregados em mucilagem, falcados a fusiformes, lisos, 1-septados, hialinos, $12-15 \times 1-1,5 \mu \mathrm{m}$; uma sétula em cada extremidade, $5,4-8,4 \mu \mathrm{m}$ compr.

Material examinado: 6.XII.2007, D.S. Santos s.n. (HUEFS 134727).

A combinação dos caracteres conídios 1-septados, uma sétula nas extremidades e presença de seta, é encontrada em quatro espécies: Dictyochaeta malaysiana Kuthub., D. novaeguineensis (Matsush.) A.I. Romero, D. tortuosa (B. Sutton) Whitton, McKenzie \& K.D. Hyde e Dictyochaeta anamorfo de Chaetosphaeria dingleyae (Kuthubutheen \& Nawawi 1991a). Dictyochaeta malaysiana e D. novae-guineensis possuem conídios mais largos (3-4 $\mu \mathrm{m}$ e 2,53,5 $\mu \mathrm{m}$, respectivamente) e D. tortuosa apresenta setas tortuosas (Kuthubutheen \& Nawawi 1991a; Whitton et al. 2000). As características do material examinado estão de acordo com Hughes \& Kendrick (1968) e Whitton et al. (2000). Esta é a primeira referência da espécie para a América do Sul. Registros dessa espécie na Austrália (Whitton et al. 2000);
Estados Unidos da América (Raabe et al. 1981); México (Abarca et al. 2004); Nova Zelândia (Hughes \& Kendrick 1968).

Dictyochaeta anamorfo de Chaetosphaeria pulchriseta S. Hughes, W.B. Kendr. \& Shoemaker, N.Z. Jl Bot. 6: 356. 1968.

Fig. 1 i-k

Setas eretas, retas ou ligeiramente flexuosas, septadas, lisas, castanhas, penúltima célula geralmente castanho-escura, 168-330 × 5-7 $\mu \mathrm{m}$; conidióforos macronemáticos, mononemáticos, solitários ou em grupos de 2-5 ao redor da seta, eretos, retos ou flexuosos, septados, lisos, castanhos a castanho-claros, 25,5-45 × 3,6-5 $\mu \mathrm{m}$; células conidiogênicas polifialídicas, com até três proliferações percurrentes, integradas, terminais, com colaretes; conídios 0-septados, agrupados em mucilagem, falcados a fusiformes, ligeiramente curvos, gutulados, lisos, hialinos, 15-25 × 1,8-4 $\mu \mathrm{m}$, com uma sétula em cada extremidade, $1,8-3 \mu \mathrm{m}$ compr. Material examinado: 24.XII.2008, T.S. Santa Izabel s.n. (HUEFS 155085); 8.VI.2008, T.S. Santa Izabel s.n. (HUEFS 155086); 21.X.2008, T.S. Santa Izabel s.n. (HUEFS 155087); 24.XII.2008, T.S. Santa Izabel s.n. (HUEFS 155088); 23.V.2008, T.S. Santa Izabel s.n. (HUEFS 155089); 7.I.2008, T.S. Santa Izabel s.n. (HUEFS 155090).

Setas com a penúltima célula castanho-escura e conídios multigutulados estão presentes em Dictyochaeta vittata Kuthub. \& Nawawi e $D$. intermedia Gusmão \& Leão-Ferreira (Cruz et al. 2008). Dictyochaeta anamorfo de Chaetosphaeria pulchriseta difere das espécies relacionadas pelas sétulas menores. O material examinado está de acordo com Kuthubutheen \& Nawawi (1991b) e Holubová-Jechová (1984). Este constitui o primeiro registro da espécie para América do Sul. Espécie anteriormente registrada em Brunei, China (Whitton et al. 2000); antiga Checoslováquia, Cuba (Holubová-Jechová 1984); Estados Unidos da América, Malásia (Kuthubutheen \& Nawawi 1991b); Nova Zelândia (Hughes \& Kendrick 1968).

Dictyochaeta matsushimae (Hewings \& J.L. Crane) Whitton, McKenzie \& K.D. Hyde, Fungal Diversity 4: 140. 2000. Bas.: Codinaea matsushimae Hewings \& J.L. Crane, Mycotaxon 13(2): 423. 1981.

Fig. $11-n$

Setas solitárias, eretas, retas ou flexuosas, lisas, castanho-escuras na base, castanho-claras no ápice, 300-360 × 4,5-6 $\mu \mathrm{m}$; conidióforos macronemáticos, mononemáticos, eretos, retos ou flexuosos, simples, lisos, castanho-claros, 40,5-66 
$\times 4,5 \mu \mathrm{m}$; células conidiogênicas mono ou polifialídicas, determinadas, integradas, lageniformes, lisas, subhialinas, 25,5-34,5 ×2 $\mu \mathrm{m}$; conídios falcados a alantóides, 3-septados, hialinos, 21-25,5 ×3 $\mu \mathrm{m}$; uma sétula em cada extremidade, $9-12 \mu \mathrm{m}$ compr.

Material examinado: 29.VIII.2008, T.S. Santa Izabel s.n. (HUEFS 134724); 17.V.2008, T.S. Santa Izabel s.n. (HUEFS 155084).

Conídios 3-septados e com sétulas nas extremidades são observados em Dictyochaeta matsushimae, D. caatingae A.C. Cruz \& Gusmão, D. macrospora Kuthub. \& Nawawi e D. triseptata (Matsush.) R.F. Castañeda. Destas, D. macrospora e $D$. triseptata não apresentam setas. A presença de seta é observada em Dictyochaeta caantigae, a qual se diferencia de D. matsushimae pelos conídios maiores e sétulas inconspícuas (Castañeda-Ruiz 1986; Kuthubutheen \& Nawawi 1991a; Cruz et al. 2008). O material examinado está de acordo com a descrição de Hewings \& Crane (1981). Este constitui o primeiro registro para o Neotrópico. Distribui-se nos Estados Unidos da América (Hewings \& Crane 1981) e na antiga União Soviética (Farr \& Rossman 2009).

Endophragmiella boothii (M.B. Ellis) S. Hughes, N.Z. Jl Bot. 17(2): 147. 1979. Bas.: Endophragmia boothii M.B. Ellis, Mycol. Pap. 72: 35. 1959.

Fig. 1 o-q

Conidióforos macronemáticos, mononemáticos, solitários ou em grupos, eretos, retos ou levemente flexuosos, 4-6 septados, simples, lisos, castanhos, 92-189 ×4 $\mu \mathrm{m}$; células conidiogênicas terminais, cilíndricas, ápice truncado, lisas, 2-4 proliferações percurrentes, castanho-claras, 3-4 $\mu$ m larg.; conídios solitários, secos, obpiriformes, 3-septados, lisos, lúmen celular reduzido, castanhos a castanhoclaros, $18-22,5 \times 9 \mu \mathrm{m}$; base truncada, $2 \mu \mathrm{m}$ larg. Secessão rexolítica.

Material examinado: 17.V.2008, T.S. Santa Izabel s.n. (HUEFS155091); 28.VII.2008, D.S. Santos s.n. (HUEFS 148831).

Endophragmiella B. Sutton foi proposto com a espécie-tipo E. pallescens B. Sutton, para acomodar espécies anteriormente incluídas em Endophragmia Duvernoy \& Maire que apresentam conídios com secessão rexolítica e células conidiogênicas com proliferações percurrentes (Hughes 1979; CastañedaRuiz et al. 1995). Endophragmiella boothii difere das demais espécies do gênero pelos conídios triseptados com o lúmen da célula reduzido (Wu \& Zhuang 2005). $\mathrm{O}$ material examinado está de acordo com $\mathrm{Wu} \&$
Zhuang (2005), porém, apresentou conídios menores que os descritos por Ellis (1959). Este constitui o primeiro registro para o Neotrópico. Além destes registros para China (Wu \& Zhuang 2005); Escócia (Farr \& Rossman 2009); Estados Unidos da América (como Endophragmia boothii, Sutton 1978); Inglaterra (como Endophragmia boothii, Ellis 1971); Nova Zelândia (Hughes 1979).

Endophragmiella pallescens B. Sutton, Mycol. Pap. 132: 62. 1973.

Fig. 2 a-d

Conidióforos macronemáticos, mononemáticos, solitários ou em grupos, eretos, retos ou flexuosos, septados, simples, lisos, castanhos, castanhoclaros no ápice, $50-150 \times 4,5-6,5 \mu \mathrm{m}$; células conidiogênicas terminais, cilíndricas, ápice truncado, lisas, com proliferações percurrentes; conídios solitários, secos, elipsóides, célula basal maior que a apical, 1-septados, lisos, castanhos, $12-16 \times 4,5-7,5 \mu \mathrm{m}$. Secessão rexolítica.

Material examinado: 15.X.2008, T.S. Santa Izabel s.n. (HUEFS 155092).

Endophragmiella pallescens assemelha-se a E. uniseptata (M.B. Ellis) S. Hughes pelos conídios 1-septados, elipisóides com célula basal maior que a apical. No entanto, E. uniseptata possui conídios maiores (Hughes 1979). O material examinado difere dos apresentados por Sutton (1973) e HolubováJechová (1986) no que se refere à ramificação dos conidióforos e na presença de conídios 1-2-septado, porém, está de acordo com Wu \& Zhuang (2005). Este é o primeiro registro para o Brasil. Anteriormente registro para Argentina (Romero \& Pildain 2003); Canadá (Sutton 1973); antiga Checoslováquia (Holubová-Jechová 1986); China (Wu \& Zhuang 2005); Inglaterra (Farr \& Rossman 2009).

Eversia parvula Hol.-Jech., Èeská Mykol. 41(1): 31. 1987.

Fig. $2 \mathrm{e}-\mathrm{f}$

Conidioma em esporodóquio. Conidióforos semi-macronemáticos a macronemáticos, mononemáticos, retos ou flexuosos, simples ou ramificados, septados, castanho-claros, 40-60 ×3$4 \mu \mathrm{m}$. Células conidiogênicas holoblásticas, integradas, terminais, cilíndricas, com proliferações percurrentes; conídios solitários, oblongos, secos, aplanados, muriformes, lisos, formados por dois braços unidos, célula basal subhialina, castanhoclaros a castanhos, $18-24 \times 7.5-10,5 \mu \mathrm{m}$.

Material examinado: 9.VI.2008, T.S. Santa Izabel s.n. (HUEFS 155094); 13.X.2008, D.S. Santos s.n. (HUEFS 148832). 
Eversia J.L. Crane \& Schokn. é constituído por duas espécies, E. parvula e E. subopaca (Cooke $\&$ Ellis) Crane \& Schokn. que se diferenciam pelo número de braços no conídio. E. subopaca possui 3-4 braços enquanto $E$. parvula possui 2 braços. (Holubová-Jechová 1987). O material examinado apresentou conidióforos maiores e conídios mais largos que os descritos por Holubová-Jechová (1987) e Abarca et al. (2004). Não foi observada a formação de anelações conspícuas castanhoescuras na região apical dos conidióforos; entretanto, as demais características do material examinado possibilitaram o enquadramento do espécime em E. parvula. Esta é a primeira referência da espécie para a América do Sul. Registros para Cuba (Holubová-Jechová 1987); México (Abarca et al. 2004); antiga União Soviética (Melnik 2000).

Gyrothrix hughesii Piroz., Mycol. Pap. 84: 22. 1962.

Fig. 2 g-i

Setas eretas, septadas, 3-5 ramificações verticiladas, lisas, castanhas, 82,5-105 × 2-3 $\mu \mathrm{m}$; conidióforos ausentes; células conidiogênicas poliblásticas, anelídicas, evidentes, surgindo lateralmente à superfície da hifa somática ou da base da seta, oboclavadas a lageniformes, subhialinas 6-7,5 ×3-4,5 $\mu \mathrm{m}$; conídios cilíndricos a fusiformes, arranjados numa fina camada esbranquiçada na base da seta, hialinos, $13,5-15 \times 1,5-2 \mu \mathrm{m}$.

Material examinado: T.S. Santa Izabel s.n., 7.VIII.08 (HUEFS 155098).

Gyrothrix foi proposto por Corda em 1884 (apud Pirozynski 1962) e é caracterizado por apresentar setas ramificadas, lisas ou verrucosas, conidióforos ausentes, células conidiogênicas poliblásticas, lageniformes a subuladas, subhialinas a hialinas e conídios falcados, cilíndricos ou fusiformes. Circinotrichum possui características semelhantes à Gyrothrix diferindo deste por apresentar setas não ramificadas (Pirozynski 1962). Gyrothrix hughesii é semelhante a G. inops (Berlese) Piroz. e G. ramosa Zucconi \& Onofri pela presença de setas verticiladas (Pirozynski 1962; Zucconi \& Onofri 1989). Gyrothrix inops diferencia-se das espécies mencionadas pelas setas maiores, levemente verrucosas e com ramificações opostas (Pirozynski 1962). Gyrothrix ramosa possui setas verrucosas maiores, com ápice curvo e filiforme (Zucconi \& Onofri 1989). Este constitui o primeiro registro para a América do Sul. Anteriormente com registros para Cuba (Mercado-Sierra \& Mena-Portales 1995); Gana, Serra Leoa, Sudão, (Farr \& Rossman 2009); Índia (Pirozinski \& Patil 1970); Paquistão (Pirozinski 1962).
Helicoubisia coronata Lunghini \& Rambelli, Micol. Ital. 8(1): 21. 1979.

Fig. $2 \mathrm{j}-1$

Conidióforos macronemáticos, mononemáticos, solitários, eretos, retos, simples, septados, lisos, castanho-escuros, 63-107 × 4-6 $\mu \mathrm{m}$; ápice inflado com quatro células conidiogênicas em verticilo; células conidiogênicas holoblásticas, evidentes, denticuladas, terminais, determinadas, cuneiformes, lisas, castanho-claras, 3,5-4 × 2,5-3 $\mu \mathrm{m}$; conídios solitários, lisos, secos, enrolados 1 vez, 6-8 septados, simples, castanho-claros a subhialinos, 8-11 $\mu \mathrm{m}$ diâm.; largura do filamento 2,5-4 $\mu \mathrm{m}$.

Material examinado: 25.VII.2008, D.S. Santos s.n. (HUEFS 137808).

O gênero monotípico Helicoubisia foi introduzido por Lunghini \& Rambelli (1979) com a espécie-tipo $H$. coronata. Matsushima (1993) propôs uma nova espécie para o gênero Moorella P. Rag. Rao \& D. Rao, M. monocephala Matsush., que apresentava as mesmas características de $H$. coronata. Pinnoi et al. (2004), analisando esses dois gêneros, propôs a sinonimização de $M$. monocephala com $H$. coronata. O material examinado está de acordo com o descrito por Lunghini \& Rambelli (1979), exceto pela largura do filamento do conídio, que no material examinado possui dimensões maiores. Esta representa a primeira ocorrência da espécie para o Brasil. Espécie presente também na China (Lu et al. 2000); Costa do Marfim (Lunghini \& Rambelli 1979); Equador e Peru (como Moorella monocephala; Matsushima 1993); Índia (Vittal \& Dorai 1995); Malásia (Kuthubutheen \& Nawawi 1994); Tailândia (Pinnoi et al. 2006).

Minimelanolocus navicularis (R.F. Castañeda) R.F. Castañeda, Cryptog. Mycol. 22(1): 9. 2001. Bas.: Pseudospiropes navicularis R.F. Castañeda, Fungi Cubenses II (La Habana): 10. $1987 . \quad$ Fig. 2 m-o

Conidióforos macronemáticos, mononemáticos, simples, solitários ou em grupos, eretos, retos ou flexuosos, septados, lisos, castanhos, castanho-claros no ápice, 45-155 × 4-5 $\mu \mathrm{m}$; células conidiogênicas poliblásticas, integradas, terminais, simpodiais, lócus conidiogênico inconspícuo; conídios solitários, naviculados, secos, lisos, 3-septados, raramente 2 -septados, célula basal e apical subhialinas a castanho-claras, células centrais castanho-escuras, $18-23 \times 6,5-8,0 \mu \mathrm{m}$.

Material examinado: 28.VII.2008, T.S. Santa Izabel s.n. (HUEFS 155099); 6.X.2008, T.S. Santa Izabel s.n. (HUEFS 155100). 


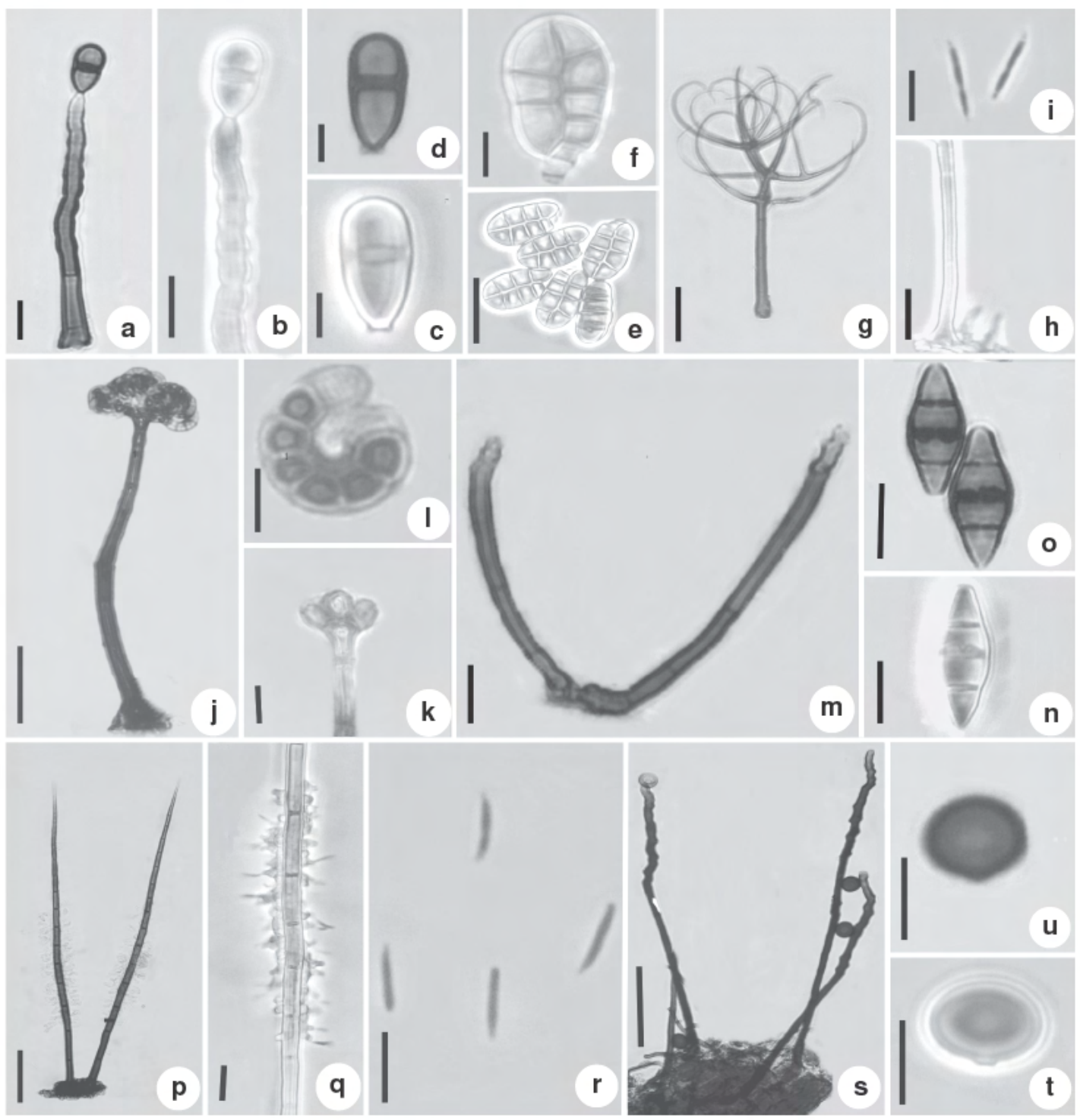

Figura 2 - a-d. Endophragmiella pallescens B. Sutton - a. aspecto geral; b. detalhe das proliferações percurrentes (seta); c-d. conídios. e-f. Eversia parvula Hol.-Jech - e. conídios; f. conídio. g-i. Gyrothrix hughesii Piroz - g. seta; h. detalhe das células conidiogênicas; i. conídios. j-1. Helicoubisia coronata Lunghini \& Rambelli -j. aspecto geral; k. detalhe das células conidiogênicas; 1 . conídio. m-o. Minimelanolocus navicularis (R.F. Castañeda) R.F. Castañeda - m. conidíoforos; $n$. conídio; o. conídios. p-r. Selenodriella ponmudiensis (Varghese \& V.G. Rao) R.F. Castañeda \& Saikawa - p. aspecto geral; q. detalhe das células conidiogênicas; r. conídios. s-u. Virgariella atra S. Hughes - s. aspecto geral; t-u. conídios. Barra $=50 \mu$ (p, s); $20 \mu$ (e, g, j, m); $10 \mu$ (a, b, h, i, n, o, q, r, t, u); $5 \mu$ (c, d, f, k, l).

Figure 2 - a-d. Endophragmiella pallescens B. Sutton - a. general aspect; b. detail of percurrent proliferations (arrow); c-d. conidia. e-f. Eversia parvula Hol.-Jech - e. conidia; f. conidium. g-i. Gyrothrix hughesi Piroz - g. setae; h. detail of conidiogenous cells; i. conidia. $\mathrm{j}-1$. Helicoubisia coronata Lunghini \& Rambelli - j. general aspect; $\mathrm{k}$. detail of conidiogenous cells; 1 . conidium. $\mathrm{m}-\mathrm{o}$. Minimelanolocus navicularis (R.F. Castañeda) R.F. Castañeda - $\mathrm{m}$. conidiophores; $\mathrm{n}$. conidium; o. conidia. p-r. Selenodriella ponmudiensis (Varghese \& V.G. Rao) R.F. Castañeda \& Saikawa - p. general aspect; q. detail of conidiogenous cells; r. conidia. s-u. Virgariella atra S. Hughes - s. general aspect; t-u. conidia. Bar $=50 \mu(\mathrm{p}, \mathrm{s}) ; 20 \mu(\mathrm{e}, \mathrm{g}, \mathrm{j}, \mathrm{m}) ; 10 \mu(\mathrm{a}, \mathrm{b}, \mathrm{h}, \mathrm{i}, \mathrm{n}, \mathrm{o}, \mathrm{q}, \mathrm{r}, \mathrm{t}, \mathrm{u}) ; 5 \mu(\mathrm{c}$, $\mathrm{d}, \mathrm{f}, \mathrm{k}, \mathrm{l})$. 
Minimelanolocus R.F. Castañeda \& Heredia foi estabelecido com a espécie-tipo M. navicularis, para acomodar espécies anteriormente incluídas em Pseudospiropes M.B. Ellis, que apresentam conídios euseptados, células conidiogênicas poliblásticas, integradas, com proliferações simpodiais, holoblásticas, raramente enteroblásticas e com lócus conidiogênico inconspícuo ou com uma pequena proeminência (Castañeda-Ruiz et al. 2001). O gênero é composto atualmente por 18 espécies (Ma et. al. 2008; Zhang et al. 2009). Minemelanolocus navicularis difere das demais espécies do gênero pelos conídios naviculados (Castañeda-Ruiz 1987). O material examinado apresenta conidióforos maiores que os apresentados por Castañeda-Ruiz (1987). Esta espécie é reportada pela primeira vez para a América do Sul. Anteriormente registros para Cuba (Castañeda-Ruiz 1987).

Selenodriella ponmudiensis (Varghese \& V.G. Rao) R.F. Castañeda \& Saikawa, Mycotaxon 85: 225. 2003. Bas.: Circinotrichum ponmudiensis Varghese \& V.G. Rao, Bot. Notiser 131(2): 215. 1978.

Fig. 2 p-r

Conidióforos setiformes, macronemáticos, mononemáticos, simples, eretos, retos ou flexuosos, septados, castanhos, castanho-claros no ápice,

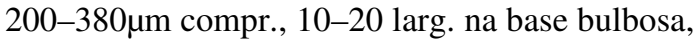
1,5-2,5 larg. no ápice; células conidiogênicas poliblásticas, surgindo diretamente do conidióforo ou de células suportes, ampuliformes a lageniformes, na região mediana do conidióforo, castanho-claras a subhialinas, 3,0-4,5 × 1,5-3,0 $\mu \mathrm{m}$; região fértil, $75-150 \mu \mathrm{m}$; conídios clavados a alantóides, lisos, 0 -septados, hialinos, agregados em mucilagem 7,2-10,8 $\times 1,2-1,5 \mu \mathrm{m}$.

Material examinado: 31.I.2008, T.S. Santa Izabel s.n. (HUEFS 155096); 16.II.2009, T.S. Santa Izabel s.n. (HUEFS 155097).

Dentre as espécies de Selenodriella R.F. Castañeda \& W.B. Kendr., apenas S. ponmudiensis $e$ S. inaequilaterospora R.F. Castañeda \& W.B. Kendr. possuem células conidiogênicas dispostas apenas lateralmente. No entanto, $S$. inaequilaterospora diferencia-se de $S$. ponmudiensis pelas células conidiogênicas lageniformes a subuladas, além de conídios maiores, fusiformes a vermiformes (Castañeda-Ruiz \& Kendrick 1991). O material examinado está de acordo com a descrição apresentada por Varghese \& Rao (1978). Este é o primeiro registro da espécie para o Brasil. Registro dessa espécie também em Cuba (como $C$. ponmudiensis, Castañeda-Ruiz et al. 1997a); Índia (como C. ponmudiensis, Varghese \& Rao 1978); Venezuela (Castaneda-Ruiz et al. 2003).

Virgariella atra S. Hughes, Can. J. Bot. 31(5): 654. 1953.

Fig. $2 \mathrm{~s}-\mathrm{u}$

Conidióforos macronemáticos, mononemáticos, simples, eretos, retos ou flexuosos, septados, lisos, castanho-escuros, 180-270 × 4,5-6 $\mu \mathrm{m}$; células conidiogênicas poliblásticas, integradas, terminais, cilíndricas, simpodiais; conídios solitários, secos, 0 -septados, elipsóides, subglobosos a ovais, lisos, castanho-escuros, 10,5-12 × 10,5-13 $\mu \mathrm{m}$.

Material examinado: 11.VI.2008, T.S. Santa Izabel s.n. (HUEFS 155093).

São aceitas oito espécies em Virgariella S. Hughes (Delgado-Rodrigues \& Mena-Portles 2003). O gênero é caracterizado pelos conidióforos macronemáticos, mononemáticos, simples, com células conidiogênicas poliblásticas, integradas, terminais, simpodiais, cilíndricas e conídios solitários, secos, fusiformes a elipsóides, subesféricos, esféricos, globosos, subglobosos, lisos ou verrucosos, castanhos (Hughes 1953; Ellis 1971; Sutton 1992; Delgado-Rodrígues \& Mena-Portales 2003). Virgariella atra está mais relacionada a $V$. globigera (Sacc. \& Ellis) S. Hughes; no entanto esta espécie apresenta conídios globosos a subglobosos e menores (Delgado-Rodrígues \& Mena-Portales 2003). O material examinado apresentou conidióforos maiores e conídios menores do que os descritos por Hughes (1953). Este constitui um novo registro para o continente americano. Anteriormente apenas China (Farr \& Rossman 2009); Inglaterra (Hughes 1953).

Outras espécies de fungos conidiais encontradas no município de Morro do Chapéu, Bahia:

Actinocladium rhodosporum Ehrenb., Jahrb. Gewächsk. 1(2): 52. 1819. (HUEFS 155193).

Atrosetaphiale flagelliformis Matsush., Matsush. Mycol. Mem. 8: 14. 1995. (HUEFS 155194).

Beltrania rhombica Penz., Michelia 2 (no. 8): 474. 1882. (HUEFS 155148).

Beltraniella portoricensis (F. Stevens) Piroz. \& S.D. Patil, Can. J. Bot. 48(3): 575. 1970. (HUEFS 15515).

Brachysporiella gayana Bat., Bol Secr. Agric. (Pernambuco) 19(1-2): 109. 1952. (HUEFS 155226).

Brachysporiellina fecunda S.M. Leão, Gusmão, R.F. Castañeda \& A.C. Cruz, Mycotaxon 104: 310. 2008. (HUEFS 155161).

Chalara affinis Sacc. \& Berl., Atti Inst. Veneto Sci. lett., ed Arti, Sér. 6 3: 741. 1885. (HUEFS 155188).

Chalara alabamensis Morgan-Jones \& E.G. Ingram, Mycotaxon 4(2): 489. 1976. (HUEFS 155189). 
Chloridium virescens var. virescens (Pers.) W. Gams \& Hol.-Jech., Stud. Mycol. 13: 17. 1976. (HUEFS 155162).

Chloridium transvaalense Morgan-Jones, R.C. Sinclair \& Eicker, Mycotaxon 17: 301. 1983. (HUEFS 155163). Circinotrichum falcatisporum Piroz., Mycol. Pap. 84:7. 1962. (HUEFS 155223).

Circinotrichum maculiforme Nees, Syst. Pilze (Würzburg): 19. 1816. (HUEFS 155224).

Circinotrichum olivaceum (Speg.) Piroz., Mycol. Pap. 84: 6. 1962. (HUEFS 15522).

Cordana musae (Zimm.) Höhn., Zentbl. Bakt. ParasitKde, Abt. II 2 (60): 7. 1923. (HUEFS 155190).

Cryptophiale kakombensis Piroz., Can. J. Bot. 46: 1124. 1968. (HUEFS 155198).

Cryptophiale udagawae Piroz. \& Ichinoe, Can. J. Bot. 46:1126. 1968. (HUEFS 155201).

Cryptophialoidea fasciculata Kuthub. \& Nawawi, Mycol. Res. 98 (6): 686. 1994. (HUEFS 155196).

Cryptohialoidea ramosa G. Delgado, J. Mena \& Gené, Fungal Diversity 20: 31. 2005. (HUEFS 155206)

Dactylaria candidula (Höhn.) G.C. Bhatt \& W.B. Kendr, Can. J. Bot. 46: 1256 1968. (HUEFS 155234).

Dactlaria cazorlii Mercado, Gené \& Guarro, in Gené, Mercado-Sierra \& Guarro, Mycol. Res.104 (11): 1404. 2000. (HUEFS 155230).

Dendrypiopsis atra (Corda) S. Hughes, Can. J. Bot. 31: 655. 1953. (HUEFS 155183).

Dictyochaeta novae-guineensis (Matsush.) A.I. Romero, Boln Soc. argent. Bot. 22: 76. 1983. (HUEFS 155142).

Dictyochaeta simplex (S. Hughes \& W.B. Kendr.) Hol.Jech., Folia geobot. phytotax. 19: 434. 1984. (HUEFS 155143).

Dischloridium inaequiseptatum (Matsush.) Hol.-Jech., Èeská Mykol. 41(2): 111. 1987. (HUEFS 155187).

Drechslera rostrata (Drechsler) M.J. Richardson \& E.M. Fraser, Trans. Br. mycol. Soc. 51: 148. 1968. (HUEFS 155235).

Ellisembia adscendens (Berk.) Subram., Proc. Indian natn Sci. Acad., Part B. Biol. Sci. 58(4): 183. 1992. (HUEFS 155127).

Ellisembia brachypus (Ellis \& Everh.) Subram., Proc. Indian natn Sci. Acad., Part B. Biol. Sci. 58: 183. 1992.(HUEFS 155133).

Ellisembia vaga (Nees \& T. Nees) Subram., Proc. Indian natn Sci. Acad., Part B. Biol. Sci. 58(4): 184.1992. (HUEFS 155104).

Exserticlava vasiformis (Matsush.) S. Hughes, N.Z. J1 Bot. 16(3): 332. 1978. (HUEFS 155172).

Fusariella obstipa (Pollack) S. Hughes, Mycol. Pap. 28: 9. 1949. (HUEFS 155216).

Gonytrchum chlamydosporium var. simile W. Gams \& Hol.-Jech., Stud. Mycol. 13: 88. 1976. (HUEFS 155166). Gonytrichum macrocladum (Sacc.) S. Hughes, Trans. Br. mycol. Soc. 34: 565. 1951. (HUEFS 155171).

Gyrothrix microsperma (Höhn.) Piroz., Mycol. Pap. 84:14. 1962. (HUEFS 155217).

Helicosporium gracile (Morgan) Linder, Ann. Mo. bot. Gdn 16: 281. 1929. (HUEFS 155208).
Helicosporium griseum Berk. \& M.A. Curtis, Grevillea 3 (XXVI): 51. 1874. (HUEFS 155215).

Helicosporium pannosum (Berk. \& M.A. Curtis) R.T. Moore, Mycologia 49: 582. 1957. (HUEFS 155214).

Helicsporium vesiculiferum A.C. Cruz \& Gusmão, Mycotaxon 110: 55. 2009. (HUEFS 141556).

Henicospra coronata B. Sutton \& P.M. Kir, Trans. Br. mycol. Soc. 75(2): 249. 1980. (HUEFS 155182).

Idriella setiformis R.F. Castañeda \& G.R.W. Arnold. Revta. Jardín Bot. Nac., Univ. Habana 6: 50. 1985.(HUEFS 155144). Junewangia globulosa (Tóth) W.A. Baker \& MorganJones, Mycotaxon 81: 308. 2002. (HUEFS 155231).

Memnoniella echinata (Rivolta) Galloway, Trans. Br. Mycol. Soc. 18(2): 165. 1933. (HUEFS 155180).

Menisporopsis novae-zelandiae S. Hughes \& W.B. Kendr., N.Z. J1 Bot. 6: 369. 1968. (HUEFS 155233).

Menisporopsis theobromae S. Hughes, Mycol. Pap. 48:59. 1952. (HUEFS 155232).

Myrmecridium schulzeri var. schulzeri (Sacc.) Arzanlou, W. Gams \& Crous, Stud. Mycol. 58: 84. 2007. (HUEFS 155260).

Neojohnstonia minima Gusmão \& Grandi, Mycotaxon 80: 98. 2001. (HUEFS 133851).

Paliphora inflata Gusmão, Marques \& D.A.C. Almeida, Mycologia 100(2): 306. 2008. (HUEFS 155175).

Pappimyces hastatus B. Sutton \& Hodges, Nova Hedwigia 26(2-3): 528. 1975. (HUEFS 155225).

Paraceratocladium silvestre R.F. Castañeda, Fungi Cubenses II (La Habana) 2: 9. 1987. (HUEFS 155207).

Periconia cookei E.W. Mason \& M.B. Ellis, Mycol. Pap. 56:72. 1953. (HUEFS 155213).

Phaeostalagmus tenuissimus (Corda) W. Gams \& Hol.Jech., Stud. Mycol. 13: 93. 1976. (HUEFS 155202).

Phaeoisria infrafertilis B. Sutton \& Hodges, Nova Hedwigia 27(1-2): 219. 1976. (HUEFS 155205).

Ramichloridium anceps (Sacc. \& Ellis) de Hoog, Stud. Mycol. 15: 59. 1977. (HUEFS 155165).

Speiropsis scopiformis Kuthub. \& Nawawi, Trans. Br. mycol. Soc. 89(4): 584. 1987. (HUEFS 155186).

Speiropsis pedatospora Tubaki, J. Hattori bot. Lab. 20: 171 1958. (HUEFS 155184).

Sporidesmiella aspera Kuthub. \& Nawawi, Mycol. Res. 97(11): 1305.1993. (HUEFS 155136).

Sporidesmiella cuneiformis (B. Sutton) P.M. Kirk, Trans. Br. mycol. Soc. 79(3): 481. 1982. (HUEFS 155140). Sporiesmiopsis zhejiangensis Wongsawas, H.K. Wang, K.D. Hyde \& F.C. Lin, J. Zhejiang Univ., Sci. B, 9: 798. 2008. (HUEFS 155101).

Sporidesmium tropicale var. tropicale M.B. Ellis, Mycol. Pap. 70: 58. 1958. (HUEFS 155141).

Stachybotrys chartarum (Ehrenb.) S. Hughes, Can. J. Bot. 36: 812. 1958. (HUEFS 155176).

Stachybotrys longispora Matsush. Icon. microfung. Matsush. lect. (Kobe): 145. 1975. (HUEFS 155178).

Stachybotrys parvispora S. Hughes, Mycol. Pap. 48:74. 1952. (HUEFS 155261).

Thozetella cristata Piroz. \& Hodges, Can. J. Bot. 51(1): 168. 1973. (HUEFS 133854). 
Thozetella queenslandica Paulus, P.Gadek \& K.D. Hyde, Mycologia 96: 1081. 2004. (HUEFS 155212).

Umbellidion radulans B. Sutton \& Hodges, Nova Hedwigia 26(2-3): 532. 1975. (HUEFS 155203).

Vermiculariopsiella falcata Nawawi, Kuthub. \& B. Sutton, Mycotaxon 37: 175. 1990. (HUEFS 133855).

Virgaria nigra (Link) Nees, Nat. Arr. Brit. Pl. (London) 1: 553. 1817. (HUEFS 155210).

Volutella minima Höhn., Sber. Akad. Wiss. Wien, Math.naturw. Kl., Abt. 1, 118: 1543. 1909. (HUEFS 155262).

Wiesneriomyces laurinus (Tassi) P.M. Kirk, Trans. Br. mycol. Soc. 82(4): 748. 1984. (HUEFS 155181).

Zanclospora brevispora var. brevispora S. Hughes \& W.B. Kendr., N.Z. J1 Bot. 3: 156. 1965. (HUEFS 155160).

Zanclospora novae-zelandiae S. Hughes \& W.B. Kendr., N.Z. J1 Bot. 3: 152. 1965. (HUEFS 155155).

\section{Agradecimentos}

Os autores agradecem ao Programa de PósGraduação em Botânica - PPGBot/UEFS e ao PPBIO (Programa de pesquisa de biodiversidade no Semiárido). T. S. Santa Izabel e L.F.P. Gusmão agradecem ao CNPq (proc. 474589/2008-0). D.A.C. Almeida e D.A. Santos agradecem a CAPES e a FAPESB, respectivamente.

\section{Referências}

Andrade-Lima, D. 1981. The caatinga dominium. Revista Brasileira de Botânica 4: 149-153.

Abarca, G.H.; Estebanez, M.R.; Mota, R.M.A.; Portales, J.M. \& Mercado-Sierra, A. 2004. Adiciones al conocimiento de la diversidad de los hongos conidiales del bosque mesófilo de montaña del estado de Veracruz. Acta Botánica Mexicana 66: 1-22.

Barbosa, F.R.; Gusmão, L.F.P.; Castañeda-Ruiz, R.F.; Marques, M.F.O. \& Maia, L.C. 2007. Conidial fungi from the semi-arid Caatinga biome of Brazil. New species Deightoniella rugosa \& Diplocladiella cornitumida with new records for the neotropics. Mycotaxon 102: 39-49.

CABI Bioscience Database. 2010. Index fungorum. Disponível em <http://www.indexfungorum.org>. Acesso em 2 jan 2010.

Castañeda-Ruiz, R.F. 1986. Fungi cubense. Instituto de Investigaciones Fundamentales em Agricultura Tropical, La Habana.

Castañeda-Ruiz, R.F. 1987. Fungi vubenses 2. Instituto de Investigaciones Fundamentales en Agricultura Tropical, La Habana.

Castañeda-Ruiz, R.F. 2005. Metodología en el estúdio de los hongos anamorfos. In Anais do V Congresso Latino Americano de Micologia, Brasília. Pp.182-183.

Castañeda-Ruiz, R.F.; Guarro, J. \& Cano, J. 1995. Notes on conidial fungi. II. A new species of Endophragmiella. Mycotaxon 54: 403-406.
Castañeda-Ruiz, R.F.; Guarro, J. \& Cano, J. 1997a. Notes on conidial fungi. XII. New or interesting hyphomycetes from Cuba. Mycotaxon 63: 169-181.

Castañeda-Ruiz, R.F.; Gusmão, L.F.P.; Abarca, G.H. \& Saikawa, M. 2006. Some hyphomycetes from Brazil. Two new species of Brachydesmiella, two new combinations for Repetophragma, and new records. Mycotaxon 95: 261-270.

Castañeda-Ruiz, R.F.; Heredia, G.; Reyes, M.; Arias, R.M. \& Decock, C. 2001. A revision of the genus Pseudospiropes and some new taxa. Cryptogamie, Mycologie 22: 1-18.

Castañeda-Ruiz, R.F.; Iturriaga, T.; Minter, D.W.; Saikawa, M.; Vidal, G. \& Velazquez-Noa, S. 2003. Microfungi from Venezuela. A new species of Brachydesmiella, a new combination, and new records. Mycotaxon 85: 211-229.

Castañeda-Ruiz, R.F. \& Kendrick, W.B. 1991. Ninetynine conidial fungi from Cuba and three from Canada. University of Waterloo Biology Series 35: 1-32.

Castañeda-Ruiz, R.F.; Kendrick, W.B. \& Guarro, J. 1997b. Notes on conidial fungi. XIV. New hyphomycetes from Cuba. Mycotaxon 65: 93-106.

Cruz, A.C.R.; Gusmão, L.F.P. \& Castañeda-Ruiz, R.F. 2007a. Conidial fungi from the semi-arid Caatinga biome of Brazil. Subramaniomyces pulcher sp. nov. and notes on Sporidesmium circinophorum. Mycotaxon 102: 25-32.

Cruz, A.C.R.; Gusmão, L.F.P.; Ferreira, S.M.L. \& Castañeda-Ruiz, R.F. 2007b. Conidial fungi from the semi-arid Caatinga biome of Brazil. Diplococcium verruculosum sp. nov. and Lobatopedis longistriatum sp. nov. Mycotaxon 102: 33-38.

Cruz, A.C.R.; Leão-Ferreira, S.M.; Barbosa, F.R. \& Gusmão, L.F.P. 2008. Conidial fungi from semiarid Caatinga biome of Brazil. New and interesting Dictyochaeta species. Mycotaxon 106: 15-27.

Cruz, A.C.R.; Santa Izabel, T. S.; Leão-Ferreira, S.M. \& Gusmão, L.F.P. 2009. Conidial fungi from the semiarid Caatinga biome of Brazil. New species and new records of Helicosporium. Mycotaxon 110: 53-64.

Delgado-Rodríguez, G. \& Mena-Portales, J. 2003. Virgariella ellipsospora sp.nov. (Hyphomycetes, anamophic fungi) from Cuba. Cryptogamie, Mycologie 24: 153-157.

Ellis, M.B. 1959. Clasterosporium and some allied dematiaceae - Phragmosporae. II. Mycological Papers 72: 1-75.

Ellis, M.B. 1971. Dematiaceous hyphomycetes. Commonwealth Mycological Institute, Kew.

Farr, D.F. \& Rossman, A.Y. Fungal databases, systematic mycology and microbiology laboratory, ARS, USDA. Disponível em < http://nt.ars-grin.gov/ fungaldatabases $>$. Acesso em 31 jul 2009.

Gusmão, L.F.P.; Barbosa, F.R. \& Barbosa, F.F. 2006. Fungos conidiais. In: Gusmão, L.F.P. \& Maia, L.C. 
(eds.). Diversidade e caracterização dos fungos do semi-árido brasileiro. Ministério da Ciência e Tecnologia, Associação Plantas do Nordeste, Recife. Pp. 161-201

Gusmão, L. F. P.; Leão-Ferreira, S.M; Marques, M.F.O. \& Almeida, D.A.C. 2008. New species and records of Paliphora from the Brazilian semi-arid region. Mycologia 100: 306-309.

Hewings, A.D. \& Crane, J.L. 1981. The genus Codinaea. Three new species from the Americas. Mycotaxon 13: 419-427.

Holubová-Jechová, V. 1984. Lignicolous hyphomycetes from Czechoslovakia. 7. Chalara, Exochalara, Fusichalara and Dictyochaeta. Folia geobotánica et Phytotaxonomica 19: 387-438.

Holubová-Jechová, V. 1986. Lignicolous Hyphomycetes from Czechoslovakia. 8. Endophragmiella and Phragmocephala. Folia geobotánica et Phytotaxonomica 21: 173-197.

Holubová-Jechová, V. 1987. Studies on hyphomycetes from Cuba V. Six new species of dematiaceous hyphomycetes from Havana Province. Èeská Mykologie 41: 29-36.

Hughes, S.J. 1953. Conidiophores, conidia and classification Conidiophores, conidia and classification. Canadian Journal of Botany 31: 577-659.

Hughes, S.J. 1979. Relocation of species of Endophragmia auct. with notes on relevant generic names. New Zealand Journal of Botany 17: 139-188.

Hughes, S.J. \& Kendrick, W.B. 1968. New Zealand fungi. 12. Menispora, Codinaea, Menisporopsis. New Zealand Journal of Botany 6: 323-375.

Junqueira, M.E.R \& Bianchini, R.S. 2006. O gênero Evolvulus L. (Convolvulaceae) no município de Morro do Chapéu, BA, Brasil. Acta Botanica Brasilica 20: 157-172.

Kuthubutheen, A.J. \& Nawawi, A. 1991a. Key to Dictyochaeta and Codinaea species. Mycological Research 95: 1224-1229.

Kuthubutheen, A.J. \& Nawawi, A. 1991b. Eight new species of Dictyochaeta (Hyphomycetes) from Malaysia. Mycological Research 95: 1211-1219.

Kuthubutheen, A.J. \& Nawawi, A. 1994. Henicospora longissima sp. nov., Obeliospora triappendiculata sp. nov., Paraulocladiumfabisporum sp. nov. and other hyphomycetes from Malaysia. Mycological Research 98: 677-685.

Leão-Ferreira, S.M.; Cruz, A.C.R.; Castañeda-Ruiz, R.F. \& Gusmão, L.F.P. 2008. Conidial fungi from the semi-arid Caatinga biome of Brazil. Brachysporiellina fecunda sp. nov. and some new records for Neotropica. Mycotaxon 104: 309-312.

Lu, B.; Hyde, K.D.; Ho, W.H.; Tsui, K.M.; Taylor, J.E.; Wong, K.M. \& Yanna \& Zhou, D. 2000. Checklist of Hong Kong fungi. Fungal Diversity Research Series 5: 1-207.
Lunghini, D. \& Rambelli, A. 1979. Helicoubisia e Talekpea, due nuovi generi di ifali demaziacei. Micologia Italiana 8: 21-24.

Ma, J.; Zhang, K. \& Zhang, X.G. 2008. Two new species of the genus Minimelanolocus in China. Mycotaxon 104: 147-151.

Maia, L.C. \& Gibertoni, T.B. 2002. Fungos registrados no semi-árido nordestino. In: Sampaio, E.V.S.B.; Giulietti, A.M.; Virgínio, J. \& Rojas, C.F.L.G. (eds.). Vegetação e flora da caatinga. Associação Plantas do Nordeste, APNE/CNIP, Recife. Pp.163-176.

Marques, M.F.O.; Barbosa, F.R.; Gusmão, L.F.P.; Castañeda Ruiz, R.F. \& Maia, L.C. 2007. Conidial fungi from the semi-arid Caatinga biome of Brazil. Cubasina microspora sp. nov., a note on C. albofusca, and some new records for South America. Mycotaxon 102: 17-23.

Matsushima, T. 1993. Matsushima Mycological Memoirs n. 7. Published by the author, Kobe.

Maury, C.M. (org.). 2002. Biodiversidade brasileira Avaliação e identificação de áreas e ações prioritárias para conservação, utilização sustentável e repartição de benefícios da biodiversidade nos biomas brasileiros. Ministério do Meio Ambiente, Brasília. 340p.

Melnik, V.A. 2000. Definitorium fungorum Rossiae. Classis Hyphomycetes. Fasc. 1. Fam. Dematiaceae. Nauka, San Petersburgo. 370p.

Mercado-Sierra, A. \& Mena-Portales, J. 1995. Hifomicetes dematiáceos de tres provincias orientales de Cuba. Revista Iberoamericana de Micología 12: 101-107.

Morgan-Jones, G., Sinclair, R.C. \& Eicker, A. 1983. Notes on Hyphomycetes. XLIV. New and rare Dematiaceous species from the Transvaal. Mycotaxon 17: 301-316.

Pinnoi, A.; Lumyong, S.; Hyde, K.D. \& Jones, E.B. G. 2006. Biodiversity of fungi on the palm Eleiodoxa conferta in Sirindhorn peat swamp forest, Narathiwat, Thailand. Fungal Diversity 22: 205-218.

Pinnoi, A.; Pinruan, U.; Hyde, K.D. \& Lumyong, S. 2004. Submersisphaeria palmae sp. nov. and key to genus and notes on Helicoubisia. Sydowia 56: 72-78.

Pirozynski, K.A. 1962. Circinotrichum and Gyrothix. Mycological Papers 84: 1-28.

Pirozynski, K.A. \& Patil, S.D. 1970. Some setose Hyphomycetes of leaf litter in south India. Canadian Journal of Botany 48: 567-581.

Raabe, R.D.; Conners, I.L. \& Martinez, A.P. 1981. Checklist of plant diseases in Hawaii. Institute of Tropical Agriculture and Human Resources, University of Hawaii, Honolulu. Information Text Series 22. 313p.

Romero, A.I. \& Pildain, M.B. 2003. Contribución al estudio de los hongos xilófilos de la Argentina. VII. Deuteromycotina en Eucalyptus viminalis (Myrtaceae). Boletín de la Sociedad Micológica de Madrid 28: 125-134.

Sutton, B.C. 1973. Hyphomycetes from Manitoba and Saskatchewan, Canada. Mycological Papers 132: 1-143. 
Sutton, B.C. 1978. New and interesting Hyphomycetes from Tampa, Florida. Mycologia 70: 784-801.

Sutton, B.C. 1992. The relationships of Virgariella verrucosa sp. nov. on Aloe leaves from Turkey. Sydowia 44: 321-331.

Varghese, K.I.M \& Rao, V.G. 1978. Two new setose hyphomycetes from India. Botaniska Notiser 131: 215-217.

Velloso, A.L.; Sampaio, E.V.S.B. \& Pareyn, F.G.C. 2002. Ecorregiões propostas para o bioma caatinga. Associação Plantas do Nordeste - APNE, Instituto de Conservação Ambiental, The Nature Conservancy do Brasil, Recife. 76p.

Vittal, B.P.R. \& Dorai, M. 1995. Studies on litter fungi VIII. Quantitative studies of the mycoflora colonizing Eucalyptus tereticornis Sm. litter. Kavaka 22/23: 35-41.

Whitton, S.R.; Mckenzie, E.H.C. \& Hyde, K.D. 2000. Dictyochaeta and Dictyochaetopsis species from the Pandanaceae. Fungal Diversity 4: 133-158.

Wu, W. \& Zhuang, W. 2005. Sporidesmium, Endophragmiella and related genera from China. Fungal Diversity Research Series 15. Fungal Diversity Press, Hong Kong. 351p.

Zhang, K.; Fu, H.B. \& Zhang, X.G. 2009. Taxonomic studies of Minimelanolocus from Yunnan, China. Mycotaxon 109: 95-101.

Zucconi, L. \& Onofri, S. 1989. Gyrothrix ramosa sp. nov. and notes on Gyrothrix citricola. Mycological Research 92: 380-382. 